In breast cancer radiotherapy, the internal mammary lymphatic chain is treated in the target volume in a group of patients with high risk criteria. There are a number of different techniques in breast radiotherapy because of the variability of the anatomic region, structures and risk criteria in the irradiation field. When irradiating the target volume we also consider homogeneity of dose distribution and minimizing the dose to critical structures such as the heart and lung. In this study, we have evaluated the dose distribution of different radiotherapy techniques in twelve patients with left breast cancer who had breast conserving surgery or mastectomy. A twodimensional computerized planning system (2-DCPS) was used for each patient to compare wide-field, oblique photonelectron, perpendicular photon-electron and oblique-electron techniques in terms of dose homogeneities in the target volume, the doses received by the heart and lung, and the coverage of the internal mammary chain. Critical structures were irradiated with acceptable dose percentages besides the internal mammary chain with both wide-field, photon-electron and oblique-electron techniques. The wide-field technique was easy to perform and exposed the heart to a smaller radiation dose than photonelectron techniques. The oblique electron techniques provide a minimal radiation dose to critical structures. In oblique electron techniques, if the internal mammary chain was not covered in the target volume, the heart dose was minimized. In conclusion, we suggest using obliqueelectron techniques in breast irradiation where the internal mammary is in the target volume.

Key words: breast cancer, radiotherapy planning and techniques, computerassisted.

\section{Research on different techniques in breast cancer radiotherapy}

\author{
Mehmet Hakan Dogan ${ }^{1}$, Seyit Burhanedtin Zincircioglu' ${ }^{1}$, Mahmut Aydinol $^{2}$
}

1Department of Radiation Oncology, School of Medicine, Dicle University, Diyarbakır, Turkey

2Department of Physics, Faculty of Science, Dicle University, Diyarbakır, Turkey

\section{Introduction}

Breast cancer is the most common cancer type in women. Its annual incidence increases as the age gets older. It constitutes approximately $30 \%$ of the malign diseases which occur commonly in women and $16 \%$ of deaths due to cancer in women. Because of the success of mammography in early diagnosis and the advances in chemotherapy and radiotherapy, the treatment of breast cancer varies frequently and includes many treatment combinations. Breast conserving therapy (BCT) is the current surgical standard treatment in breast cancer and modified radical mastectomy/radical mastectomy, which is another optimal treatment in appropriate cases, includes full dissection of the breast, musculus pectoralis major and minor and the axilla. However, its unacceptable outcomes in terms of cosmetics and function have brought modified radical mastectomy forward featuring preservation of the musculus pectoralis major [1]. Radiotherapy is absolutely administered after breast preserving surgery. Axillary dissection treats axillary metastatic lymph nodes. The status of the lymph nodes as a result of axillary dissection is important in determining the indication and intensity of adjuvant chemotherapy [2]. In patients with diffuse axillary lymph node metastasis, radiotherapy to the axillary region is administered additionally. Radiotherapy to the mammaria interna (MI) lymph node chain is administered in patients with high risk. Lymphoscintigraphy shows that high drainage is present in MI lymph nodes in breast cancer localized in the middle and internal quadrants even in early stages [3, 4]. In radiotherapy for breast cancer, different techniques can be used because of the anatomical variance of the region and depending on whether $\mathrm{Ml}$ is included in the target volume. These include standard technique, wide area (WA) technique, photon-electron (PE) technique and oblique electron (OE) technique. Studies have shown that radiotherapy decreases local-regional recurrence rates from $33 \%$ to $9 \%$ and below $[5,6]$. However, there are also some studies which have shown that the benefit of radiotherapy in breast cancer treatment is balanced with early and late toxicity [7, 8]. The authors who recommended radiation of MI lymph nodes aimed to perform the therapy with the best technique. As stated in many publications, cardiac tissue is exposed to some degree of radiation in radiotherapy of the left breast and chest wall and consequently a small increase in cardiac deaths is observed in the long term [9-11]. Use of wedge filters, collimation system, dynamic multileaf collimator and three-dimensional tomography-based planning system is recommended to provide a homogeneous distribution of dose in the target volume, to decrease the dose administered to the heart and lung and to decrease cutaneous reactions [12-16]. Other than these radiotherapy techniques, intensity-modulated radiotherapy (IMRT), helical tomotherapy and proton beam treatment provide better dose reductions for critical organs [17].

Among these techniques, WA technique and PE technique include MI lymph node radiation. In WA technique, tangential photon areas are used in a way 
that $\mathrm{MI}$ is included. PE technique can be administered in three ways: oblique photon-electron (OPE) technique, vertical photon-electron (VPE) technique and OE technique. In OPE technique, photon-electron beams are applied to the $\mathrm{MI}$ area obliquely in parallel with the tangential area. In VPE technique, they are applied vertically to the chest wall while the gantry angle is 0 degrees. In OE technique, the electron beam is applied in parallel with the tangential area including the lateral point of the breast. The breast is treated by using the medial and lateral tangential photon areas. Use of a CT-based three-dimensional planning system has decreased the risk of cardiac death by $50 \%$ excluding an important amount of normal tissue (heart, lung) outside the radiation area [18]. There are studies which have reported that the lung volume exposed to radiation in OPE technique is decreased compared to the standard and WA techniques and a non-homogeneous dose distribution is obtained in the volume exposed to radiation in WA technique, since the width of the lateral area is increased [19]. Arthur et al. [20] emphasized the problem arising from the depth of $\mathrm{MI}$ lymph node and recommended use of multiple sections with computed tomography (CT) providing the target volume to be kept inside the reference equal dose. In patients with large breast volume, dose inhomogeneity is high inside the breast volume; there are studies which have shown that $10 \%$ of the breast volume is equal to or higher than $110 \%$ of the defined dose [21-23].

The aim of this study was to calculate the dose homogeneity inside the target volume, the doses received by the breast vol-
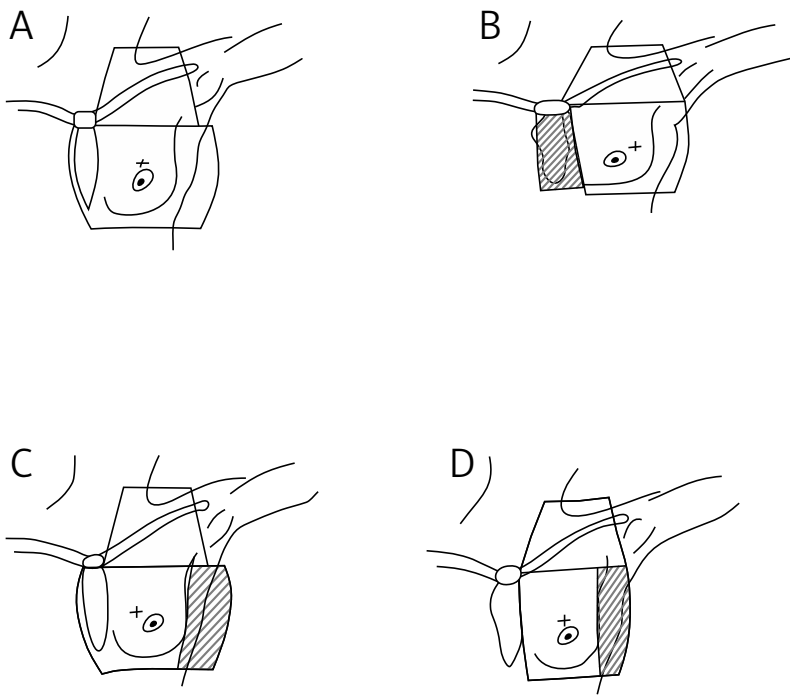

Fig. 1. Treatment areas on the patient's surface. In all three techniques, SSD technique was used so that the localization of the reference point remained the same in all set-ups. A) In WA technique, the edge of the internal tangential areas was opened more broadly including MI. B) In PE technique, the internal and external tangential areas of the breast were treated. The edges of the oblique or vertical electron areas opened for MI were combined with the edge of the internal tangential area. C) In OE technique where $\mathrm{MI}$ is included in the target volume, the lateral point region was taken in parallel with the oblique electron area and the external tangential area. D) In OE technique where $\mathrm{MI}$ is not included in the target area, the lateral point region was taken in parallel with the oblique electron area and the external tangential area umes and the doses of the critical organs (heart, lung) and MI. The techniques to be compared were determined to be WA, OPE, VPE and OE techniques. 12 female patients with left breast cancer who had undergone breast preserving surgery were included in our study. The fact that the patients had left breast cancer provided cardiac dose analyses to be performed. Treatment techniques were compared in all patients. The accuracy of the treatment planning system was checked using an ion chamber in a solid water phantom.

\section{Material and methods}

\section{Patients}

Patients with left breast cancer who had undergone direct surgery (mastectomy) or breast preservation surgery were included in our study. A total of 12 patients were treated in Dicle University, School of Medicine, Department of Radiation Oncology. The mean age of the patients was 55, ranging between 33 and 65 years. Patients with right breast cancer were excluded to evaluate the cardiac dose.

\section{Computed tomography simulation}

Computed tomography sections were taken from the chin up to the upper abdominal region with three-millimeter intervals. The images obtained from CT were used to transfer the target volume and critical organ contours onto the computerized treatment planning with a digitizer. The contours of the heart, lung, left breast, right breast and MI were determined for each section. The contour of the left breast was marked by placing a wire along CT scanning. In all patients, the posterior border of the left breast inside the target volume was selected as the rib-lung interface. The superior border was selected $3 \mathrm{~mm}$ below the skin surface. The cranial border of the target volume was determined to be the lower part of the clavicular head. The contour of the target volume planned for MI (PTV) was taken according to the localization of MI lymph nodes. This volume was extended from the first intercostal interval to the xiphoid bone craniocaudally.

Set-up was performed in all patients with the shaded surface display technique (SSD technique). Since the reference point had to be inside the patient contour, this point was placed in the center of the breast area. In all techniques, gantry angles were adjusted so that the posterior edge of the tangentials was parallel. To achieve an accurate set-up with SSD technique the collimator angle was given or 1-2 was not given. In addition, we superimposed the areas by superposing the wires placed on the area edges during simulation. In WA technique, maximum lung width was adjusted to be smaller than $3 \mathrm{~cm}$ (Fig. 1A). With the help of the view image in the direction of the beam the amount of the lung volume inside the area was determined craniocaudally. The beam edge image on the patient's surface was observed in two-dimensional planning.

$\mathrm{PE}$ techniques were simulated on the $\mathrm{CT}$ and simulator device (Fig. 1B). While the preserved breast and chest wall were planned with photon beam with SSD, a separate area in parallel with the tangential area was opened in OPE technique for MI and a separate area perpendicular to the chest wall was opened in VPE technique. The applied beam was planned as electron and photon combination. In both PE techniques, the concentric area of both photon and electron areas was 


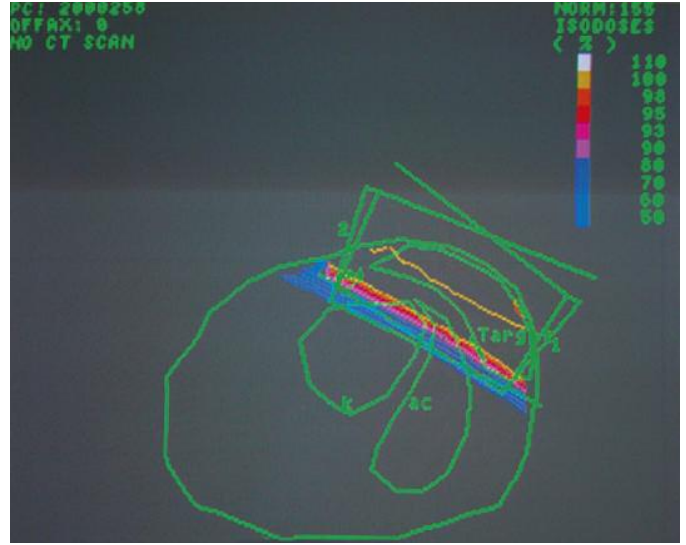

Fig. 2. Dose distribution in the WAT in a spared breast. The left breast, left lung, heart and MI contours are indicated. Different equivalent dose curves are shown

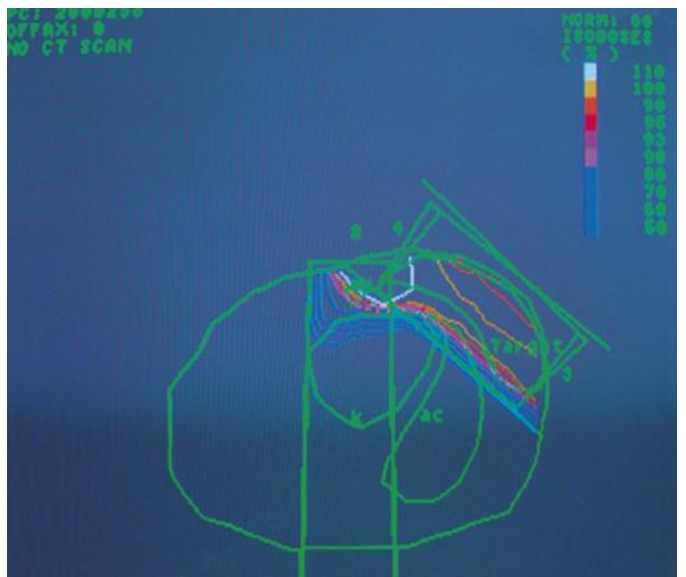

Fig. 4. Dose distribution in the VPET in a spared breast. The left breast, left lung, heart and MI contours are indicated. Different equivalent dose curves are shown

placed on the skin (SSD technique). In the tangential area, the concentric area was placed in the center of the area determined in CT sections so that the interval between the source and the skin was $80 \mathrm{~cm}$. The right lateral edge of the electron area was placed $3 \mathrm{~cm}$ laterally to the sternal middle line and the left lateral edge was combined with the edge of the internal tangential photon area on the skin surface. Generally, an area width of 5-6 cm was selected. The length of the electron area was extended so that the edges of the internal and lower quadrants were irradiated. The upper edge of the electron area and the upper edge of the tangential photon areas were at the same level. In OE techniques, the lateral point area of the breast was taken in parallel with the electron area and the tangential areas. The length of the electron area was extended as the length of the breast area (Fig. 1C, D).

Since the upper border of the tangential areas is in the same localization in all techniques in all patients, the lower border of the supraclavicular area is superposed on this upper border and the size, depth and localization of the area do not change for the supraclavicular area, the irradiated lung volume is the same. In all three techniques, CT images, organ contours, target volume and beam information were trans-

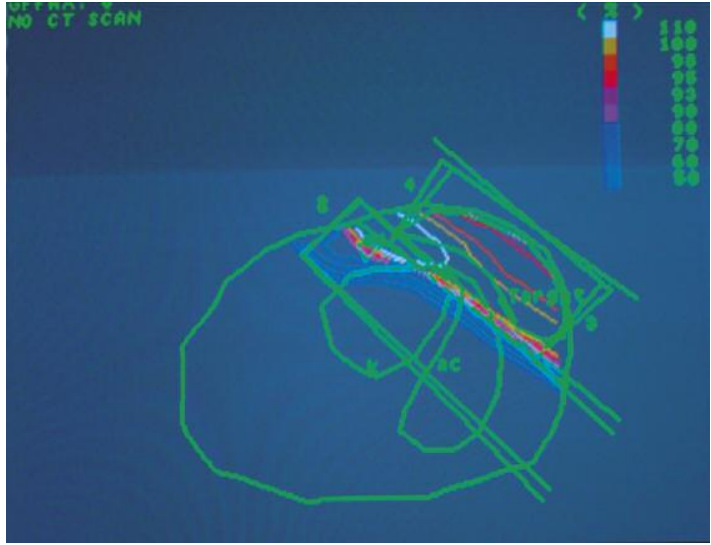

Fig. 3. Dose distribution in the OPET in a spared breast. The left breast, left lung, heart and MI contours are indicated. Different equivalent dose curves are shown

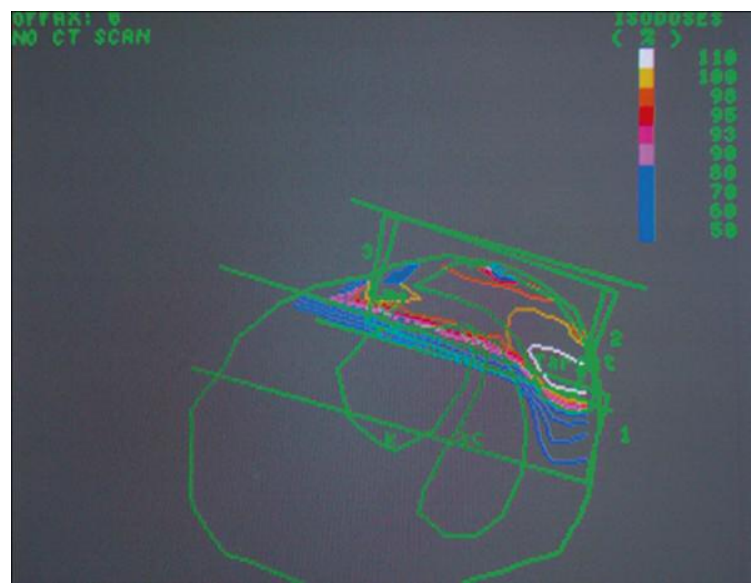

Fig. 5. Dose distribution in the $\mathrm{OE}(\mathrm{mi}+)$ technique in a spared breast. The left breast, left lung, heart and MI contours are indicated. Different equivalent dose curves are shown

ferred to the planning system using a digitizer. The Target 1.2.0(GE) system was used in two-dimensional planning and dose optimization.

In WA technique, dose distributions were optimized using a wedge filter (Fig. 2). In all plannings, lung inhomogeneity was corrected. In PE and OE techniques, optimization in electron areas was provided considering gantry angle, beam weight and the patient's width (Fig. 3-5). The contralateral breast was made to be excluded from the irradiation area by checking the edges of the tangential area with an image program in the direction of the beam. Warm areas were not more than $115 \%$ of the applied dose in beam overlap regions. The dose was planned as a total of $45 \mathrm{~Gy}$ in 25 fractions. While ${ }^{60} \mathrm{Co}$ gamma photon energy was used in all techniques, an electron beam was used only in PE and OE techniques. Since MI lymph node depth was smaller than $3 \mathrm{~cm}$, the energy was selected as $9 \mathrm{MeV}$. In PE technique, MI area was irradiated so that $2 / 5$ of the total dose was electron beam and $3 / 5$ of the total dose was photon beam. In OE techniques, the breast point region was irradiated so that the dose ratios of the electron area and the tangential photon area were equal. 


\section{Data collection and statistical analysis}

Maximum mean percentage dose values for the left breast, $\mathrm{MI}$, left lung and heart and volume percentages for different reference doses were calculated with standard deviations. Treatment techniques were compared using (\%) values of the difference of the mean values for each critical structure.

\section{Results}

\section{Left breast volume}

In all techniques, the left breast was wrapped in accordance with the defined equal dose. When the three techniques were compared with each other in terms of volume percentages of the left breast irradiated for each reference dose, no statistically significant difference was observed. These values are shown in Table 1 in detail. The localization of high dose regions was observed to change depending on the treatment technique. Warm regions were observed to form in the entries of the internal and external areas in WA technique and in the overlap region of the tangential photon area and electron area in PE and OE techniques (Figs. 3-5). In all three techniques, high dose values observed were found to be decreased as one goes from the central section to the lower and upper sections.

\section{Covering MI lymph nodes}

In WA, PE and OE techniques, MI was covered appropriately in all patients who were irradiated. When the irradiated
MI volume percentages were compared for each reference dose, they were found to be significantly higher in WA technique compared to PE and OE techniques. Values are given in Table 2. The total dose percentage was found to be $100.2 \%$ for WA technique, $85.5 \%$ for OPE technique, $90.8 \%$ for VPE technique and $88.1 \%$ for OE technique where the mammaria interna was included.

\section{Lung volume}

When the irradiated lung volume percentages were compared for each reference dose without considering the supraclavicular area, OE technique and oblique PE technique were found to be statistically different. The results are shown in Table 3. When the supraclavicular area was included in the calculation, a 10\% increase in volume percentages was found, but it was not separately included in the evaluation, since the supraclavicular area is the same in all techniques for all patients.

\section{Heart volume}

The volume percentages of the heart irradiated are given in Graphic 4.4 for the reference doses of 20, 30 and $40 \mathrm{~Gy}$. In PE techniques, the volume percentage of the irradiated heart is significantly higher compared to WA technique at the reference dose of $20 \mathrm{~Gy}$. In OE techniques, the volume percentage of the irradiated heart is significantly smaller compared to PE and WA techniques. However, at higher reference doses this was not maintained.

Table 1. When 45 Gy PTV is targeted, total dose percentage of the left breast and irradiated volume percentages for different reference doses

\begin{tabular}{|c|c|c|c|c|}
\hline \multirow[t]{2}{*}{ Technique } & \multicolumn{3}{|c|}{ Volume percentages for reference doses } & \multirow{2}{*}{$\begin{array}{l}\text { Total dose; } \\
p \text {-value (\%) }\end{array}$} \\
\hline & $45 \mathrm{~Gy}$ & $50 \mathrm{~Gy}$ & 53.5 Gy & \\
\hline VPET & $95.5 \pm 2.0$ & $55.4 \pm 1.5$ & $12.2 \pm 0.3$ & 101; NS \\
\hline WAT & $94.7 \pm 1.3$ & $55.9 \pm 0.8$ & $6.3 \pm 0.1$ & 101.6; NS \\
\hline OPET & $96.1 \pm 1.9$ & $55.7 \pm 1.3$ & $11.5 \pm 0.2$ & 101.5; NS \\
\hline $\mathrm{OE}(\mathrm{mi}+)$ & $95.8 \pm 1.7$ & $56.3 \pm 1.0$ & $12.0 \pm 0.2$ & 101.6; NS \\
\hline $\mathrm{OE}(\mathrm{mi}-)$ & $95.1 \pm 2.4$ & $55.5 \pm 1.2$ & $11.7 \pm 0.3$ & 101.5; NS \\
\hline
\end{tabular}

VPET - vertical photon-electron technique; WAT - wide area technique; OPET - oblique photon-electron technique; OE(mi+) - technique where mammaria interna area is included in the irradiated left breast volume; OE(mi-) - technique where mammaria interna area is included in the irradiated left breast volume; SD - standard deviation; NS - not significant.

Data are given as percentage \pm standard deviation. No statistically significant difference was found between the groups.

Table 2. When 45 Gy PTV is targeted, total dose percentage of mammaria interna and irradiated volume percentages for different reference doses

\begin{tabular}{|c|c|c|c|c|c|}
\hline \multirow[t]{2}{*}{ Technique } & \multicolumn{4}{|c|}{ Volume percentages for reference doses } & \multirow{2}{*}{$\begin{array}{l}\text { Total dose; } \\
\text { p-value (\%) }\end{array}$} \\
\hline & $40 \mathrm{~Gy}$ & $45 \mathrm{GY}$ & $50 \mathrm{~Gy}$ & $52.5 \mathrm{~Gy}$ & \\
\hline WAT & $98.3 \pm 2.3$ & $92.1 \pm 2.1$ & $50.3 \pm 1.1$ & $25.8 \pm 0.6$ & $100.2 ;<0.05$ \\
\hline OPET & $80.0 \pm 2.7$ & $34.9 \pm 1.2$ & $2.8 \pm 0.4$ & $0.69 \pm 0.02$ & 85.5; NS \\
\hline VPET & $84.8 \pm 2.5$ & $36.7 \pm 1.1$ & $3.6 \pm 0.1$ & $0.90 \pm 0.03$ & 90.8; NS \\
\hline $\mathrm{OE}(\mathrm{mi}+)$ & $82.1 \pm 2.7$ & $37.3 \pm 1.1$ & $3.3 \pm 0.1$ & $0.80 \pm 0.02$ & 88.1; NS \\
\hline
\end{tabular}

VPET - vertical photon-electron technique; WAT - wide area technique; OPET - oblique photon-electron technique; OE(mi+) - technique where mammaria interna area is included in the irradiated left breast volume; SD - standard deviation; NS - not significant.

Data are given as percentage \pm standard deviation. The difference between WA and PE and OE techniques was found to be statistically significant ( $p<0.05$ ). 
Table 3. When 45 Gy PTV was targeted, total dose percentage of the left lung and the irradiated volume percentages for different reference doses

\begin{tabular}{|c|c|c|c|c|}
\hline \multirow[t]{2}{*}{ Technique } & \multicolumn{3}{|c|}{ Volume percentages for reference doses } & \multirow{2}{*}{$\begin{array}{l}\text { Total dose; } \\
\text { p-value (\%) }\end{array}$} \\
\hline & $20 \mathrm{~Gy}$ & $30 \mathrm{~Gy}$ & $40 \mathrm{~Gy}$ & \\
\hline VPET & $12.0 \pm 4.9$ & $11.4 \pm 4.5$ & $10 \pm 3.9$ & 12; NS \\
\hline WAT & $12.5 \pm 4.3$ & $11 \pm 3.8$ & $10 \pm 3.6$ & $14 ;$ NS \\
\hline OPET & $19.0 \pm 3.6$ & $18 \pm 3.5$ & $16 \pm 3.4$ & $19.7 ;<0.05$ \\
\hline $\mathrm{OE}(\mathrm{mi}+)$ & $6.4 \pm 3.4$ & $6.2 \pm 3.3$ & $5.4 \pm 2.8$ & $6.6 ;<0.05$ \\
\hline $\mathrm{OE}(\mathrm{mi}-)$ & $7.1 \pm 3.6$ & $6.8 \pm 3.5$ & $6.0 \pm 3.1$ & $7.3 ;<0.05$ \\
\hline
\end{tabular}

VPET - vertical photon-electron technique; WAT - wide area technique; OPET - oblique photon-electron technique; OE(mi+) - technique where mammaria interna area is included in the irradiated left breast volume; $O E(m i-)$ - technique where mammaria interna area is included in the irradiated left breast volume; SD - standard deviation; NS - not significant.

The data are given as percentage \pm standard deviation. A statistically significant difference was found between oblique PE and OE techniques and the other techniques.

Table 4. When 45 Gy PRV was targeted, total dose percentage of the heart and their radiated volume percentages for differrent reference doses

\begin{tabular}{|c|c|c|c|c|}
\hline \multirow[t]{2}{*}{ Technique } & \multicolumn{3}{|c|}{ Volume percentages for reference doses } & \multirow{2}{*}{$\begin{array}{l}\text { Total dose; } \\
p \text {-value (\%) }\end{array}$} \\
\hline & 20 Gy & 30 Gy & 40 Gy & \\
\hline VPET & $24.2 \pm 2.6$ & $11.7 \pm 2.0$ & $6.0 \pm 1.0$ & $29.2 ;<0.05$ \\
\hline WAT & $9.7 \pm 2.8$ & $8.8 \pm 2.5$ & $7.1 \pm 2.0$ & $17.8 ;<0.05$ \\
\hline OPET & $19.8 \pm 5.8$ & $19.8 \pm 2.7$ & $4.3 \pm 1.2$ & $23.8 ;<0.05$ \\
\hline $\mathrm{OE}(\mathrm{mi}+)$ & $7.4 \pm 2.8$ & $7.4 \pm 1.3$ & $1.6 \pm 0.5$ & 8.9; NS \\
\hline $\mathrm{OE}(\mathrm{mi}-)$ & $6.2 \pm 2.5$ & $6.2 \pm 1.0$ & $1.6 \pm 0.7$ & 7.4; NS \\
\hline
\end{tabular}

VPET - vertical photon-electron technique; WAT - wide area technique; OPET-oblique photon-electron technique; OE(mit) - technique where mammaria interna area is included in the irradiated left breast volume. OE(mi-) - technique where mammaria interna area is included in the irradiated left breast volume. SD - standard deviation; NS - not significant.

The data are given as percentage \pm standard deviation. The difference between PE, OE and WA techniques was found to be statistically significant ( $p<0.05$ ).

\section{Discussion}

Radiotherapy of MI lymph nodes is not as standard as axillary and supraclavicular lymphatic radiotherapy in terms of both indication and application technique. Physicians who wish to treat MI lymph nodes should consider radiation toxicity carefully, because there are studies which show that the benefit of breast cancer radiotherapy is balanced by early and late toxicity [24-26]. Many RT techniques which include IMRT investigate the effect of increasing homogeneity in the PTV and preserving healthy tissues (toxicity decreasing effect) [27-34]. In this study, broad area technique which selects the $\mathrm{MI}$ region as the target area, oblique photon electron technique, vertical photon electron technique and oblique electron technique were compared. Because of presence of adjacent critical organs and variance of anatomical structure in breast radiotherapy, different irradiation areas are used with different gantry angles and photon electron combinations. This brings along the problem of area overlap such as warm area and cold area. Generally, problems in radiotherapy technique become prominent in obese patients and it is difficult to administer an appropriate treatment with any technique. For example, including MI is difficult in obese patients especially with PE and OE techniques, because marked reductions occur in dose values in increasing depths of MI. The depth of $\mathrm{MI}$ also varies depending on the patient and the deepest localization is at the level of the first three costae and inter- costal spaces. In some obese patients, the depth of MI has been found to be larger than $6 \mathrm{~cm}$ (Kaplan et al. 1988). Most of the lymphatic drainage of the breast occurs in $\mathrm{MI}$ lymph nodes in this upper level. In our study, inclusion of MI was found to be better in WA technique compared to PE and $\mathrm{OE}$ techniques. This was in parallel with the results of the literature [19, 20]. While Dogan et al. [35] irradiated MI with a volume percentage of $93.5 \%$ at the reference dose of 45 Gy, the mean value in our study was found to be $92.1 \%$. Van Der Laan et al. [36] found the volume of the mammaria interna irradiated to be $98.5 \%$ in the 3D planning system, $98.5 \%$ in photon IMRT and $98.6 \%$ in photon electron IMRT in their study which they performed using advanced technology devices. In our study, marked reductions were observed in MI volume irradiated at higher doses compared to the reference dose of 40 Gy with PE and OE techniques and this can be attributed to rapid energy loss of electron beams in deep tissues. In the study performed by Severin et al. [19], MI lymphatics were included in broad tangential areas, a special protection block for the lung tissue was used and the target volume received at least a 45 Gy dose in all patients. Wide area techniques adapt more easily to the change in the depths of target volume. To avoid the problems arising from the change of $\mathrm{MI}$ depth, routine use of CT sections in breast plannings and lymphoscintigraphy may be recommended. 
Irradiated left lung volume may change due to the variance of the anatomical structure and breast volume. In our study, the differences in the irradiated lung volume depend on the tangential areas and MI area, since the same supraclavicular fossa area is used in all techniques for all patients. In each five techniques where the supraclavicular fossa area is included in irradiation, an increase of $10 \%$ was observed in the irradiated left lung volume. In the study performed by Severin et al. [19], addition of the supraclavicular fossa area contributed a percentage of 10-11\% to the left lung volume irradiated. In our study, all techniques were found to be similar in terms of the left lung volume irradiated independent of the inclusion or exclusion of the supraclavicular fossa area in the irradiation.

The volume of the left lung irradiated for the reference dose value of 20 Gy was found to be $12 \%$ with vertical PE technique, $12.5 \%$ with WA technique, $19 \%$ with oblique PE technique, $6.4 \%$ with OE technique where the mammaria interna was included and $7.1 \%$ with OE technique where the mammaria interna was not included (Graphic 4.3). Dogan et al. [35] found the volume of the left lung irradiated to be $11.9 \%$ with vertical PE technique, $12.4 \%$ with WA technique and $13.2 \%$ with oblique PE technique. Georgia Nicolini et al. [37] found the mean lung volume to be $18.2 \%$ at the reference dose of 20 Gy using non-coplanar radiotherapy technique. In our study, we found this value to be $19 \%$ using oblique PE technique. In all techniques we used, the lung volume irradiated did not exceed $22 \%$. Sung Ho Moon et al. [17] found the lung volume to be $0.4 \%$ with proton beam treatment, $2.3 \%$ with IMRT, $6 \%$ with 3D-CRT and $142 \%$ with Tomo Helical at a dose of $20 \%$ of the dose defined in PTV. Among the techniques we used, the best value (6.4\%) was found with OE technique where the mammaria interna was included. When we compared all techniques in terms of the volume of irradiated lung volume, it was found that the best volume values were obtained with $\mathrm{OE}$.

Extended general survival which we hope to achieve with radiotherapy in breast cancer may be confounded by late cardiac deaths due to radiotherapy. This has been shown in some studies in the literature [9-11]. Therefore, it appears to be important to keep the cardiac dose as low as possible especially in patients with left breast cancer. Gagliardi et al. [38] defined a dose-response relationship between the cardiac dose and late complications and reported that the risk became prominent when $20 \%$ of the cardiac volume received a dose of higher than $30 \mathrm{~Gy}$. In our study, the cardiac volumes irradiated were found to be significantly larger with PE techniques compared to WA technique at the reference dose of 20 Gy. With VPE technique the cardiac volume irradiated was found to be about $24.2 \pm 2.6 \%$ at the reference dose of 20 Gy. However, the cardiac volume irradiated was found to be significantly larger with WA technique compared to PE technique at higher reference doses. This was attributed to rapid loss of electron energy in deep tissues. The cardiac volume irradiated was found to be about $10 \%$ for reference doses of 30 and 40 Gy. With OE techniques the cardiac volume irradiated was found to be below $10 \%$ at the reference dose of 20 Gy. According to these ratios, it can be concluded that the techniques used do not carry a significant risk in terms of late cardiac complications. As the use of chemotherapeutic drugs which harm the heart becomes widespread, it will be important to keep the cardiac volume irradiated at the lowest level.

Another important subject is the left breast. The mean doses received by the left breast volume did not show any difference in any technique. However, an increase of 10\% in dose was observed in the overlap region of photons and electrons in $\mathrm{OE}$ and PE techniques. No difference was found between the techniques in terms of breast volume percentages wrapped with an equal reference dose of 45 Gy. Thus, the volume dose received by critical organs is more determinative for selection of the technique rather than the volume dose received by the left breast. Takeda et al. [39] reported that volumes of the left breast wrapped with an equal dose of $95 \%$ had values of $95 \%$ or above as a result of the analysis of dose-volume histograms. In our study, these volume values were found to be about $95 \%$, which was in parallel with the literature.

In conclusion, when all factors are considered, OE technique can be accepted as a more convenient and better technique compared to PE and WA techniques, because the dose received by the heart is lower while including $\mathrm{MI}$ in an appropriate way, warm points can be observed more prominently and a marked reduction in the lung irradiated is not present. To decrease warm regions in PE and OE techniques the localization of overlap regions should be changed at every 1000 cGy. This would lead to consumption of more time and labor. WA technique seems to be more problematic compared to the standard technique in terms of planning and treatment time. Conclusively, preference of OE techniques may be appropriate in breast cancer patients in whom the $\mathrm{Ml}$ area is wished or not wished to be irradiated.

In breast radiotherapy, the results of the treatment techniques developed can be improved with new techniques or superior technological devices.

The authors declare no conflict of interest.

\section{References}

1. Aydiner A, Topuz E. Onkoloji El Kitab. Istanbul Üniversitesi Onkoloji Enstitüsü Yayinlar. Istanbul 2006; 167.

2. Perez CA, Chao CKS, Brady LW. Radyasyon Onkolojisi Tedavi Kararlar. Nobel Tip Kitap Evi. Ankara 2004; 354.

3. Buchholz TA. Internal mammary lymph nodes: to treat or not to treat. Int J Radiat Oncol Biol Phys 2000; 46: 801-3.

4. Freedman GM, Fowble BL, Nicolaou N, Sigurdson ER, Torosian MH, Boraas MC, Hoffman JP. Should internal mammary lymph nodes in breast cancer be a target for the radiation oncologist? Int I Radiat Oncol Biol Phys 2000; 46: 805-14.

5. Arriagada R, Lę MG. Adjuvant radiotherapy in breast cancer - the treatment of lymph node areas. Acta Oncologica 2000; 39: 295-305.

6. Ragaz J, Jackson SM, Le N, et al. Adjuvant radiotherapy and chemotherapy in node-positive premenopausal women with breast cancer. N Engl J Med 1997; 337: 956-62.

7. Effects of radiotherapy and surgery in early breast cancer. An overview of the randomized trials. Early Breast Cancer Trialists' Collaborative Group. N Engl I Med 1995; 333: 1444-55.

8. Van de Steene J, Soete G, Storme G. Adjuvant radiotherapy for breast cancer significantly improves overall survival: the missing link. Radiotherapy Oncol 2000; 55: 263-72. 
9. Hurkmans CW, Borger JH, Bos LJ, van der Horst A, Pieters BR, Lebesque JV, Mijnheer BJ. Cardiac and lung complication probabilities after breast cancer irradiation. Radiother Oncol 2000; 55: 145-51.

10. Hřst H, Brennhovd IO, Loeb M Postoperative radiotherapy in breast cancer - long-term results from the Oslo study. Int J Radiat Oncol Biol Phys 1986; 12: 727-32.

11. Paszat LF, Mackillop WJ, Groome PA, Boyd C, Schulze K, Holowaty E. Mortality from myocardial infarction after adjuvant radiotherapy for breast cancer in the surveillance, epidemiology, and end-results cancer registries. J Clin Oncol 1998; 16: 2625-31.

12. Carruthers LJ, Redpath AT, Kunkler IH. The use of compensators to optimize the three dimensional dose distribution in radiotherapy of the intact breast. Radiother Oncol 1999; 50: 291-300.

13. Evans PM, Donovan EM, Fenton N, et al. Practical implementation of compensators in breast radiotherapy. Radiother Oncol 1998; 49: 255-65.

14. Hong L, Hunt M, Chui C, Spirou S, Forster K, Lee H, Yahalom J, Kutcher GJ, McCormick B. Intensity-modulated tangential beam irradiation of the intact breast. Int J Radiat Oncol Biol Phys 1999; 44: 1155-64.

15. Lo YC, Yasuda G, Fitzgerald TJ, Urie MM. Intensity modulation for breast treatment using static multi-leaf collimators. Int J Radiat Biol Onco Phys 2000; 46: 187-94.

16. Chałubińska J, Spych M, Łuniewska-Bury J, Fijuth J. Practical tips for treatment of radiation-induced toxicity in patients during and after radiotherapy due to locally advanced breast cancer. Prz Menopauz 2011; 15: 118-22.

17. Moon SH, Shin $\mathrm{KH}$, Kim TH, et al. Dosimetric comparision of four different external beam partial breast irradiation techniques: Three dimensional conformal radiotherapy, intensity-modulated radiotherapy, helical tomotherapy and proton beam therapy. Radiother Oncol 2009; 90: 66-73.

18. Muren LP, Maurstad G, Hafslund R, Anker G, Dahl O. Cardiac and pulmonary doses and complication probabilities in standart and con formal tangential irradiation in conservative management of breast cancer. Radiother Oncol 2002; 62: 173-83.

19. Severin D, Connors S, Thompson H, Rathee S, Stavrev P, Hanson J. Breast radiotherapy with inclusion of internal mammary nodes: a com parison of techniques with three-dimensional planning. Int J Radiat Oncol Biol Phys 2003; 55: 633-44.

20. Arthur DW, Arnfield MR, Warwicke LA, Morris MM, Zwicker RD. Internal mammary node coverage: an investigation of presently accepted techniques. Int J Radiat Oncol Biol Phys 2000; 48: 139-46.

21. Buchholz TA, Gurgoze E, Bice WS, Prestidge BR. Dosimetric analysis of intact breast irradiation in off-axis planes. Int J Radiat Oncol Biol Phys 1997; 39: 261-7.

22. Donovan EM, Johnson U, Shentall G, Evans PM, Neal AJ, Yarnold JR. Evaluation of compensation in breast radiotherapy: a planning study using multiple static fields. Int J Radiat Biol Oncol Phys 2000; 46: 671-9.

23. Neal AJ, Torr M, Helyer S, Yarnold JR. Correlation pf breast heterogeneity with breast size using 3D CT planning and dose-volume histogram. Radiother Oncol 1995; 34: 210-8.

24. Overgaard M, Hansen PS, Overgaard J, et al. Postoperative radio therapy in high risk premenopausal women with breast cancer who receive adjuvant chemotherapy. Danish Breast Cancer Cooperative Group 82b trial. N Engl J Med 1997; 337: 949-55.

25. Overgaard M, Jensen MB, Overgaard J, et al. 1999. Postoperative radiotherapy in high-risk postmenopausal breast-cancer patients given adjuvant tamoxifen: Danish Breast Cancer Cooperative Group DBCG 82c randomised trial. Lancet 1999; 353: 1641-8.

26. Ragaz J, Jackson SM, Le N, et al. Adjuvant radiotherapy and chemotherapy in node positive premenopausal women with breast cancer. N Engl J Med 1997; 337: 956-62.

27. Lingos TI, Recht A, Vicini F, Abner A, Silver B, Harris JR. Radiation pneumonitis in breast cancer patients treated with conservative surgery and radiation therapy. Int I Radiat Biol Oncol Phys 1991; 21: 355-60.

28. Boice JD Jr, Harvey EB, Blettner M, Stovall M, Flannery JT. Cancer in the contralateral breast after radiotherapy for breast cancer. N Eng J Med 1992; 326: 781-5.

29. Baral E, Larsson LE, Mattsson B. Breast cancer following irradiation of the breast. Cancer 1997; 40: 2905-10.
30. Kaija H, Maunu P. Tangential breast irradiation with or without internal mammary chain irradiation: results of a randomized trial. Radiother Oncol 1995; 36: 172-6.

31. Gyenes G, Rutqvist LE, Liedberg A, Fornander T. Long-term cardiac morbidity and mortality in a randomized trial of pre and postoperative radiation therapy versus surgery alone in primary breast cancer. Radiother Oncol 1998; 48: 185-90.

32. Mansfield CM, Komarnicky LT, Schwartz GF, et al. Ten-year results in 1070 patients with stages I and II cancer treated by conservative surgery and radiation therapy. Cancer 1995; 75: 2328-36.

33. Scrimger RA, Connors SG, Halls SB, Starreveld AA. CT-targeted irradiation of the breast and internal mammary lymph nodes using a 5field technique. Int J Radiat Biol Oncol Phys 2000; 48: 983-9.

34. Zackrisson B, Arevärn M, Karlsson M. Optimized MLC-beam arrangements for tangential breast irradiation. Radiother Oncol 2000; 54: 209-12.

35. Dogan MH, Zincircioglu SB, Zorlu F. Comparison of various radiation therapy techniques in breast cancer where target volume includes mammaria interna region. Med Dosim 2009; 34: 42-50.

36. van der Laan HP, Korevaar EW, Dolsma WV, Maduro JH, Langendijk JA. Minimising contralateral breast dose in post-mastectomy intensity-modulated radiotherapy by incorporating conformal electron irradiation. Radiother Oncol 2010; 94: 235-40.

37. Nicolini G, Fogliata A, Cozzi L Critical appraisal of a non-coplanar technique for radiotherapy of breast minimising lung involvement. Radiother Oncol 2005; 76: 319-25.

38. Gagliardi G, Lax I, Ottolenghi A, Rutqvist LE. Long-term cardiac mortality after radiotherapy of breast cancer - application of the relative seriality model. Br J Radiol 1996; 69: 839-46.

39. Takeda A, Shigematsu N, Ikeda T, et al. Evaluation of novel modified tangential irradiation technique for breast cancer patients using dosevolume histograms. Int J Radiat Oncol Biol Phys 2004; 58: 1280-8.

\section{Address for correspondence}

\section{Seyit Burhanedtin Zincircioglu}

Department of Radiation Oncology

Dicle University

School of Medicine

21280 Diyarbakir, Turkey

e-mail: sbzoglu@yahoo.com

Submitted: 26.09 .2012

Accepted: $\quad 06.11 .2012$ 